\title{
Shock Propagation Verification on Paved Mesh Geometries
}

August 18, 2011

\section{(ـawrence Livermore National Laboratory}

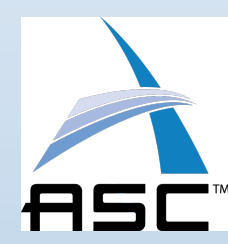




\section{DISCLAIMER}

This document was prepared as an account of work sponsored by an agency of the United States government. Neither the United States government nor Lawrence Livermore National Security, LLC, nor any of their employees makes any warranty, expressed or implied, or assumes any legal liability or responsibility for the accuracy, completeness, or usefulness of any information, apparatus, product, or process disclosed, or represents that its use would not infringe privately owned rights. Reference herein to any specific commercial product, process, or service by trade name, trademark, manufacturer, or otherwise does not necessarily constitute or imply its endorsement, recommendation, or favoring by the United States government or Lawrence Livermore National Security, LLC. The views and opinions of authors expressed herein do not necessarily state or reflect those of the United States government or Lawrence Livermore National Security, LLC, and shall not be used for advertising or product endorsement purposes.

This work performed under the auspices of the U.S. Department of Energy by Lawrence Livermore National Laboratory under Contract DE-AC52-07NA27344. 


\section{1) Introduction}

We show the results of a multi-dimensional hydrodynamic verification problem of the Kull high energy density physics (HEDP) simulation code. The ability to accurately capture the propagation of waves and shocks through material interfaces of arbitrary geometry is routinely encountered in the modeling of HEDP experiments. Constraints of computational cost in the practical application of codes such as Kull to these problems often requires careful construction of the initial mesh to concentrate mesh resolution into regions of the computational domain that are of the greatest importance. The computational mesh in such cases cannot, in general, always follow the constraint of zone-mass matching as required for optimal solution quality in von Nuemann-Richtmyer type Lagrangian hydrodynamic schemes at an acceptable computational cost. In this paper, we consider the accuracy of the Kull code in computing the propagation of a steady shock through a computational mesh that incorporates both of these properties by setting up a material interface of non-trivial geometry in a mesh that applies concentrated zoning in the vicinity of the interface. Specifically, the initial mesh has been constructed on a mesh generated using the paver tool of the Draco mesh generation package in such a manner that the initial mesh is aligned with the interface. The solution computed by the Kull code is compared against the exact analytic solution. Finally, we demonstrate the role of zone-mass mismatches in the paved mesh regions as the dominate cause of errors in the post-shock solution.

\section{2) Problem Setup}

Our test consists of a two dimensional domain that spans $0<\mathrm{x}<1$ in the horizontal direction and $-3<y<3$ in the vertical direction. It is convenient to specify the problem in dimensionless units. Our units are chosen such that the initial density and sound speed are set as $\rho=1$ and $c=1$ with a $\gamma=5 / 3$ ideal gas law equation of state, everywhere. The top boundary is set as that of an impulsively driven piston with velocity $v_{p}=-3$ in the $y$-direction. The analytic solution for the post shock density $\rho_{p s}$ and shock propagation speed $\mathrm{v}_{\mathrm{s}}$ (in units of the sound speed of the preshock gas) are given as

$$
\begin{aligned}
\frac{\rho_{p s}}{\rho} & =\frac{(\gamma+1) v_{s}^{2}}{2+(\gamma-1) v_{s}^{2}} \\
v_{s} & =\frac{v_{p}}{1-\rho / \rho_{p s}} .
\end{aligned}
$$

Our numerical solutions are advanced forward in time until the shock driven by the piston reaches $\mathrm{y}=$ -2.5 as computed by the analytic value for the shock propagation speed.

We consider three strategies for meshing the initial domain, a one dimensional case and two different two dimensional meshes. Both of the two dimensional meshing strategies include a perturbed interface that is not geometrically aligned with any of the edges of the computational domain. The perturbed interface is located at the center of the domain. The top and bottom boundaries have an isotropically spaced mesh with resolution four times coarser than the mesh spacing at the interface so that the mesh zoning is more concentrated near the interface. The initial interface separates the computational domain into two parts, a top part which spans

and a bottom part which spans

$$
A \cos (2 \pi x)<y<3
$$

$$
-3<\mathrm{y}<\mathrm{A} \cos (2 \pi \mathrm{x})
$$

The amplitude of the interface perturbation is set as $A=1 / 2$. To facilitate mesh convergence testing we specify the construction of the initial mesh in terms of a zone refinement factor, $\mathrm{R}$.

The two dimensional meshing strategies considered in this work differ in terms of how the mesh 
is generated near the interface. In the first two dimensional meshing strategy the middle section of the domain from

$$
A \cos (2 \pi x)-1 / 2<y<A \cos (2 \pi x)+1 / 2(5)
$$

is uniformly meshed along the arc-length of the cosine perturbation with

$$
\mathrm{N}_{\mathrm{M}, \mathrm{x}}=16 \mathrm{R} \operatorname{nint}(\mathrm{A} \pi)
$$

zones in the $\mathrm{x}$-direction and

$$
\mathrm{N}_{\mathrm{M}, \mathrm{y}}=8 \mathrm{R}(7)
$$

zones in the y-direction where "nint" is the nearest integer function. The top and bottom edges of the domain are set with a horizontal mesh spacing that is four times that in the middle section of the domain with

$$
\mathrm{N}_{\mathrm{T}, \mathrm{x}}=\mathrm{N}_{\mathrm{B}, \mathrm{X}}=2 \text { R. (8) }
$$

The vertical mesh spacing in the regions above and below the uniformly meshed region increases in a geometric progression as $|y|$ increases so that the horizontal and vertical mesh spacing match at middle and horizontal mesh boundaries. The Draco paver tool is used to mesh the interior of the regions above and below the uniformly meshed region in the center of the domain. An example of an initial mesh generated using this strategy is shown in the leftmost panel of figure 3. We will refer to this mesh as the "partially paved mesh". In the second meshing strategy we eliminate the uniformly meshed region in the center of the domain. The horizontal zone spacing at the top and center of the domain are the same as the first strategy and the entire computational domain is meshed using the Draco paver tool. We will refer to this mesh as the "fully paved mesh". An example of an initial mesh generated using this strategy is shown in the rightmost panel of figure 6.

In both of the two dimensional meshing strategies, the initial mesh is not zone-mass matched due to meshing some regions of the initial domain with uniform density and a geometric progression in zone spacing. To isolate the effect on the solution quality of the irregular geometry of paved mesh zones from the effect of the zone-mass mismatches we will also consider a one dimensional, shock-aligned version of the problem which dispenses with the perturbed interface in the center of the domain but uses the same geometric progression in zone length along the vertical axis as the partially paved meshing strategy.

In the following section, we compare the Kull solution against the analytic solution of the pistondriven shock propagation through the one dimensional and both two dimensional meshes using the Lagrangian, Eulerian and ALE modes of the code. The convergence properties of the numerical solution obtained with each meshing strategy is shown in terms of the domain-averaged relative error norms

$$
\begin{gathered}
L_{1} \text { relative error }=\frac{1}{A_{D}} \int\left|\frac{Q_{A}-Q_{\text {code }}}{Q_{A}}\right| d^{2} \boldsymbol{x}, \\
L_{2} \text { relative error }=\sqrt{\frac{1}{A_{D}} \int\left(\frac{Q_{A}-Q_{\text {code }}}{Q_{A}}\right)^{2} d^{2} \boldsymbol{x}}
\end{gathered}
$$

and

$$
L_{\infty} \text { relative error }=\operatorname{MAX}_{\mathbf{x}}\left|\frac{Q_{A}-Q_{\text {code }}}{Q_{A}}\right|
$$

where $\mathrm{Q}_{\mathrm{A}}$ is the fluid quantity of interest from the exact analytic solution, $\mathrm{Q}_{\text {code }}$ is the fluid quantity as computed by Kull and $A_{D}$ is the area of the computational domain. The parameters for the hydrodynamic solver have been set according to the values in Table1. The remap parameters for Eulerian and ALE modes have been set according to the values in Table 2 . The majority of these parameters are chosen to enable terms that act to better preserve energy conservation in the mesh motion strategy as this is a critical aspect for achieving the correct post shock state in purely hydrodynamic problems. In ALE mode 
the mesh is flagged for relaxation whenever any of the metrics in Table 3 fall outside of the specified range. All other input parameters to the code are left at their default values. In ALE grid relaxation is turned off at domain boundary edges. Because moving mesh boundaries are incompatible with an Eulerian approach, in Eulerian mode we have added a cap of two zones in vertical height to the top of the computational domain where we impose the analytic post-shock solution to start the shock propagation. Because the most commonly applied ALE mesh quality metrics are ill defined in one dimension, we consider only the Lagrangian and Eulerian modes in the one dimensional mesh.

\begin{tabular}{|l|l|}
\hline Parameter & Value \\
\hline Limiter & False \\
\hline HourGlassControl & 'Caramana corner pressures' \\
\hline
\end{tabular}

Table 1: Hydrodynamic solver parameters.

\begin{tabular}{|l|l|}
\hline Parameter & Value \\
\hline cornerMassAssignment & 'FlattenDelta' \\
\hline heatingOnly & False \\
\hline restoreKineticEnergy & True \\
\hline kineticEnergyThreshold & 1.0 \\
\hline enforceTotalEnergyCeiling & False \\
\hline displacementIterations & 4 \\
\hline
\end{tabular}

Table 2: Mesh remap parameters for Eulerian and ALE mode.

\begin{tabular}{|l|l|l|}
\hline Parameter & Min Value & Max Value \\
\hline skew & 0.0 & 0.85 \\
\hline taper & 0.0 & 0.5 \\
\hline oddy & 0.0 & 16.0 \\
\hline smallest angle & 30.0 & 90.0 \\
\hline
\end{tabular}

Table 3: Mesh relaxation criteria for ALE mode.

\section{3) Results}

\section{1) One Dimensional Result with Geometric Grid Spacing.}

We have found that the shock position, shock propagation speed and post shock velocity solutions given by the code are quite accurate and that the errors in the numerical solution are dominated by errors in the density. Grid scale fluctuations in the post-shock pressure smooth out quickly, in a grid zone sound crossing time. Consequently, errors in the temperature appear simply as an inverse to the error in the density. We will therefore focus on the numerical solution of the density field in evaluating the quality of the numerical solution. Figure 1 shows the density field after shock propagation through the one dimensional $\mathrm{R}=10$ mesh with geometric progression grid spacing in the Lagrangian (left) and Eulerian (right) modes. The blue curve shows the Kull solution and the black curve shows the analytic solution.

We now identify a few features of the numerical solution in the one dimensional case and we note that these features persist in a characteristically similar manner in the two dimensional meshes shown in 
the following sections. The shock discontinuity spans three computational zones and the numerical viscosity near the shock is sufficient to prevent numerical overshoots or oscillations upstream of the discontinuity. We note the so-called "wall-heating" artifact which appears as a low density spike immediately downstream of the piston at $y=-1.0$ in the Lagrangian case and $y=-1.8$ in the Eulerian case. This artifact is common to flows driven by an impulsive boundary in Lagrangian schemes for hydrodynamical flow. Two small jumps appear in the Lagrangian case in the region $-1.5<y<-2$. These errors can be attributed to the non-mass matched nature of the mesh.
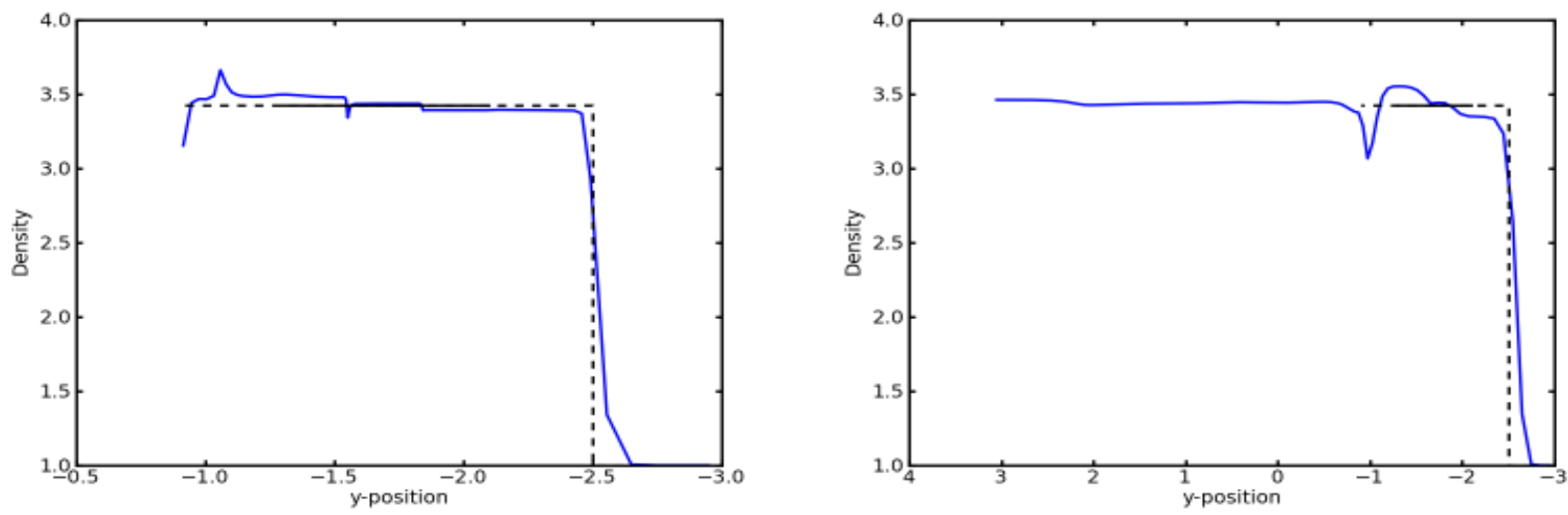

Figure 1: The density field is shown by the blue curve after shock propagation through the one dimensional $\mathrm{R}=10$ mesh with geometric progression grid spacing, in fully Lagrangian and Eulerian mode in the left and right panels, respectively. The dashed black curve shows the analytic solution while the solid portion of the black curve indicates the region for consideration in computing the $\mathrm{L}_{\infty}$ norm of the error.

Figure 2 shows the convergence of the relative error in the density field for the one dimensional mesh. In the case of the $\mathrm{L}_{\infty}$ norm we seek to quantify the solution error due to the construction of the mesh and not the wall heating error which is occurs even in perfectly mass-matched meshes. Therefore the $\mathrm{L}_{\infty}$ norm in Figure 2 and all convergence plots to follow is taken over the middle $50 \%$ of the region between the piston and the shock interface as indicated by a solid black line in Figure 1. In Lagrangian mode the $\mathrm{L}_{1}$ and $\mathrm{L}_{2}$ solution errors follow the expected first order convergence rate for discontinuous flow and the $\mathrm{L}$ follows a faster than first order convergence at coarser resolution before reaching a convergence plateau with an error of $\sim 4 \%$ due to density oscillations arising from zone mass mismatches in the initial mesh. We infer from the steady convergence of the lower order error norms that the volume occupied by these errors decreases with increasing mesh resolution but the magnitude of the errors, while small, do not converge. We will consider the effect of the magnitude of mismatches in zone mass in determining the magnitude of isolated errors such as these in section 3.3. In Eulerian mode the convergence rate of the error norms at high zone refinement factors are only slightly slower than the theoretically expected first order convergence. The solution errors in the Eulerian case are dominated by numerical diffusion. 

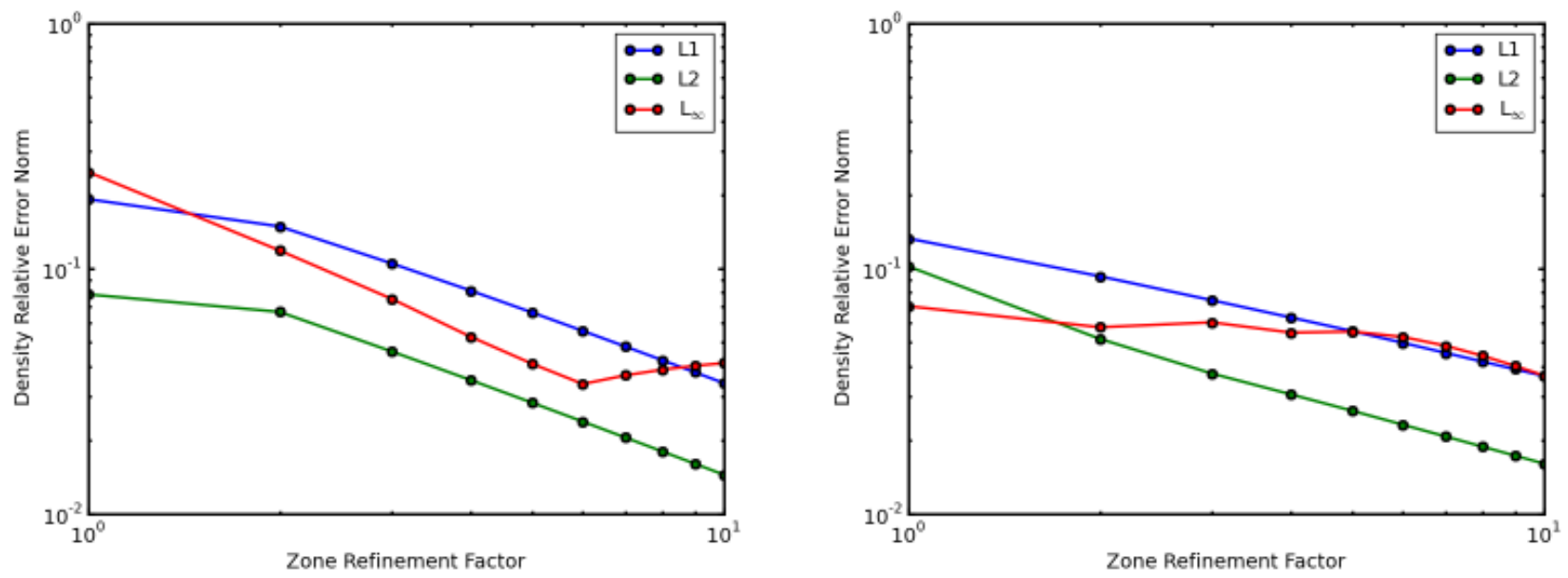

Figure 2: Convergence of the relative error in the density field for the one dimensional mesh with geometric progression grid spacing, in the Lagrangian and Eulerian mode in the left and right panels, respectively. The green curves show the $\mathrm{L}_{1}$ norm, the blue curves show the $\mathrm{L}_{2}$ norm and the red curves show the $\mathrm{L}_{\infty}$ norm.

\section{2) Two Dimensional Uniformly Gridded Interface with Paved Ends}

Figure 3 shows the density field after shock propagation through the partially paved $\mathrm{R}=2$ mesh with a uniformly meshed interface. The mesh shown for the Eulerian solution (right) is identical to the initial mesh but the Lagrangian and ALE meshes compress as the "piston zones" along the top boundary are driven downward. Because the Eulerian approach does not compress the mesh zone density following gas compression in the flow, the Eulerian solution is very diffusive, particularly for the very coarse zoning shown here. As expected, better resolution of the region of interest is achieved in the Lagrangian (left) / ALE (center) modes which exactly / approximately follow the motion of the mesh with the fluid. The shock compression flattens the isotropically zoned cosine-shaped interface region in the center of the flow by the end of the calculation and the cosine shape of the initial interface is distorted due to the varying arrival time of the planar shock along the surface of the interface. The ALE relaxation parameters were chosen to be sufficiently restrictive that no ALE relaxation occurs in the isotropically zoned central region. The solution error in the zones that would otherwise become the most badly distorted in the Lagrangian case are improved somewhat by ALE relaxation. 

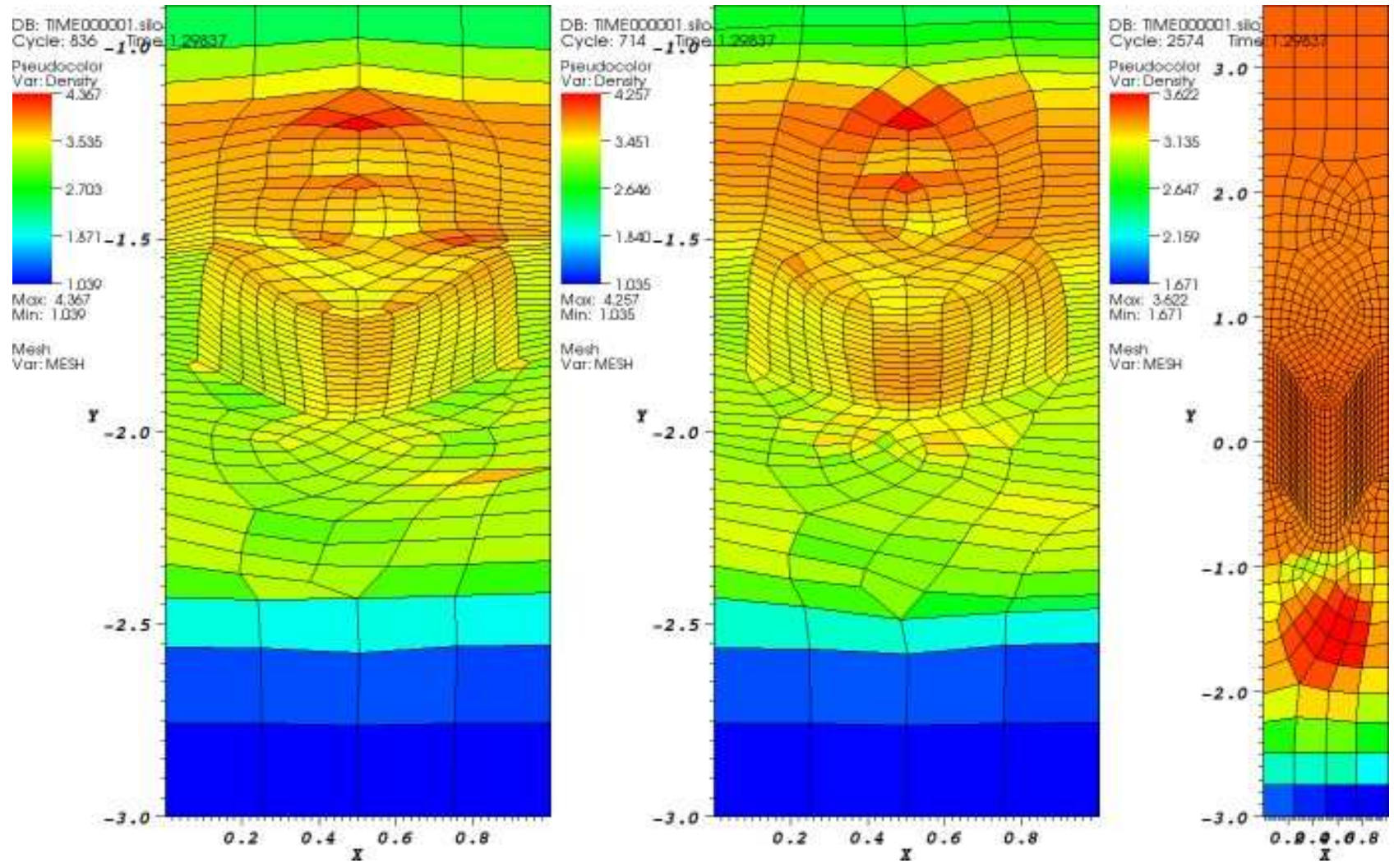

Figure 3: Density field after shock propagation through the partially paved $\mathrm{R}=2$ mesh with a uniformly meshed interface in the center in fully Lagrangian, ALE and Eulerian mode from left to right. The low resolution $\mathrm{R}=2$ mesh is shown here to accentuate the nature of the post-shock errors which are concentrated in isolated zones of high and low density regions in the post-shock flow.

Figure 4 shows one dimensional cuts of the density field after shock propagation through the partially paved $\mathrm{R}=4$ mesh with a uniformly meshed interface in the center in fully Lagrangian, ALE and Eulerian mode from left to right. The red curve indicates the maximum density, the blue curve indicates the minimum density and the green curve indicates the average density at each y-position in the domain. The black curve shows the analytic solution. The range of density spanned by the maximum and minimum of the solution at each position is generally narrower in the ALE case than the Lagrangian case owing to the improvement in solution quality in the most heavily tangled zones. 

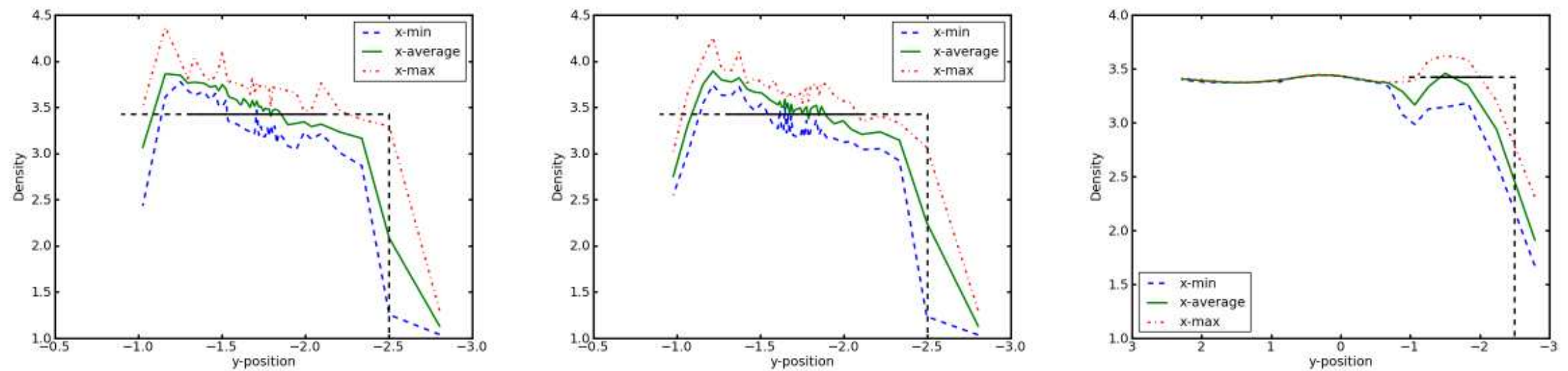

Figure 4: One dimensional cuts of the density field after shock propagation through the partially paved $\mathrm{R}=4$ mesh with a uniformly meshed interface in the center in fully Lagrangian, ALE and Eulerian mode from left to right. The red curve indicates the maximum density, the blue curve indicates the minimum density and the green curve indicates the average density at each y-position in the domain. The dashed black curve shows the analytic solution while solid portion of the black curve indicates the region for consideration in computing the $\mathrm{L}_{\infty}$ norm of the error.
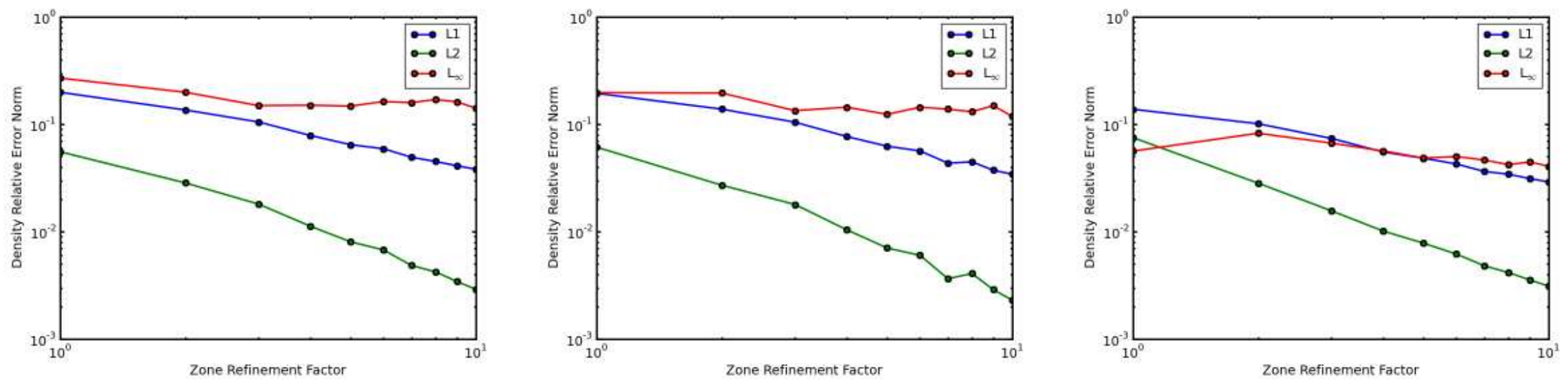

Figure 5: Convergence of the relative error in the density field for the partially paved mesh in the Lagrangian, ALE and Eulerian mode from left to right. The green curves show the $\mathrm{L}_{1}$ norm, the blue curves show the $\mathrm{L}_{2}$ norm and the red curves show the $\mathrm{L}_{\infty}$ norm. 
Figure 5 shows the convergence of the relative error in the density field for the partially paved mesh in fully Lagrangian, ALE and Eulerian mode from left to right. In contrast to the one dimensional case, the $\mathrm{L}_{2}$ convergence in all cases is better than first order and the $\mathrm{L}_{1}$ convergence is somewhat less than first order. The reason for this is that while the paver tool used to construct the mesh is constrained to apply the desired number of zones along the perimeter of the paved regions, some variation occurs on the interior so that increasing the resolution does not precisely change each zone length in the interior by the same factor. The $\mathrm{L}_{\infty}$ norm in the Eulerian case does show slow but nearly monotonic convergence, whereas the $\mathrm{L}_{\infty}$ norm in the Lagrangian and ALE cases flatten for zone refinement factors $\mathrm{R}>3$. This is consistent with the behavior due to mass mismatching noted in the one dimensional test. We note that qualitatively similar errors appear in the Eulerian case at the instant the shock discontinuity crosses the poorly mass-matched zones but that the magnitude of the errors diminish with time in the Eulerian case due to the higher degree of numerical diffusion. The errors due to mass mismatching are larger than in the one dimensional case due to variations in zone mass introduced by the paved mesh, an effect we will consider in more detail in section 3.3.

\section{3) Two Dimensional Fully Paved Mesh}

Figures 6,7 , and 8 show the fully paved mesh results corresponding to figures 3,4 and 5 of the previous section. All aspects of the solutions described in the earlier section also apply to the fully paved mesh. The only notable difference is that the $\mathrm{L}_{\infty}$ errors in the ALE and Lagrangian cases are slightly greater in the fully paved mesh case due to slight differences in the initial zone masses.
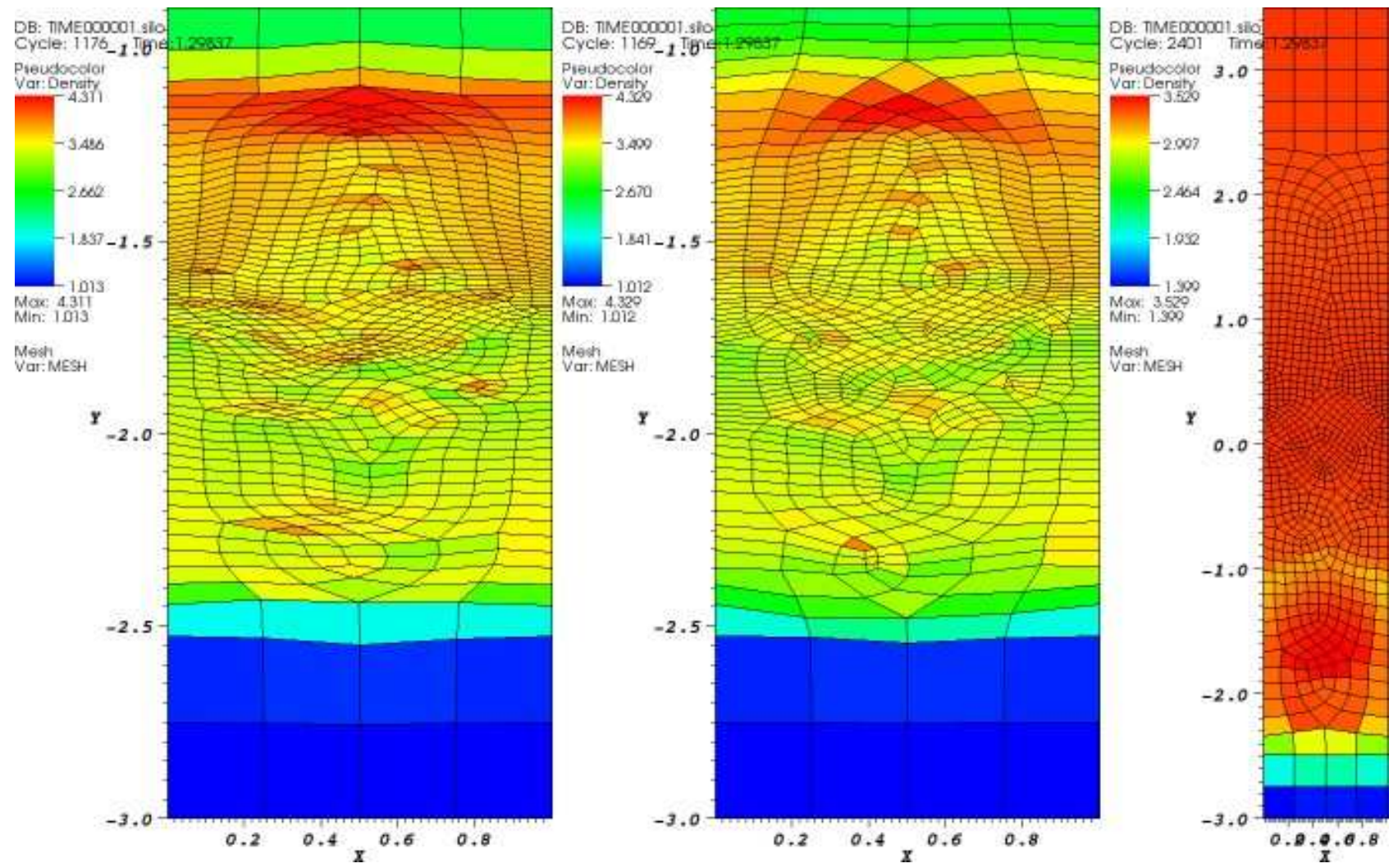

Figure 6: Density field after shock propagation through the fully paved $\mathrm{R}=2$ mesh in the center in fully Lagrangian, ALE and Eulerian mode from left to right. The low resolution $\mathrm{R}=2$ mesh is shown here to accentuate the nature of the post-shock errors which are concentrated in isolated zones of high and low density regions in the post-shock flow. 

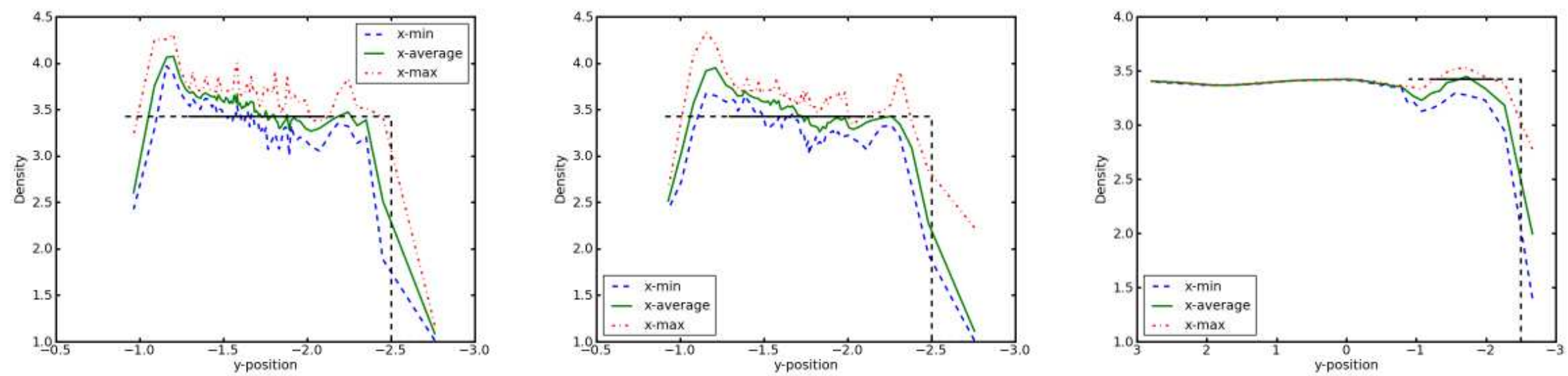

Figure 7: One dimensional cuts of the density field after shock propagation through the partially paved $\mathrm{R}=4$ mesh in fully Lagrangian, ALE and Eulerian mode from left to right. The red curve indicates the maximum density, the blue curve indicates the minimum density and the green curve indicates the average density at each y-position in the domain. The dashed black curve shows the analytic solution while the solid portion of the black curve indicates the region for consideration in computing the $\mathrm{L}_{\infty}$ norm of the error.
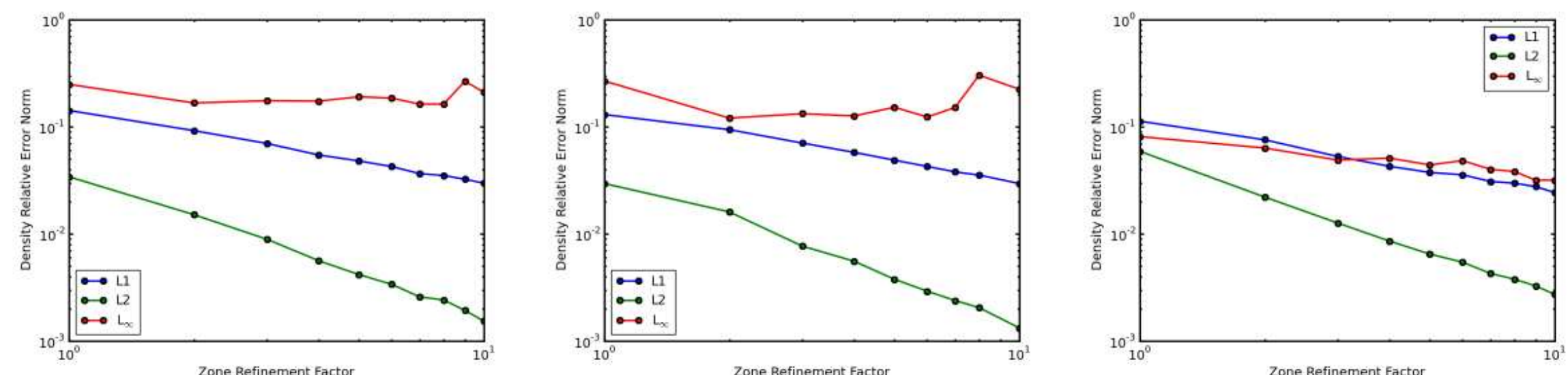

Figure 8: Convergence of the relative error in the density field for the fully paved mesh in the Lagrangian, ALE and Eulerian mode from left to right. The green curves show the $\mathrm{L}_{1}$ norm, the blue curves show the $\mathrm{L}_{2}$ norm and the red curves show the $\mathrm{L}_{\infty}$ norm. 


\section{3) The Role of Mass-Matching in One Dimension as a Function of Piston Speed}

In this section we quantify the impact of zone mass changes in the paved mesh problems by devising a test problem to isolate the impact of an isolated change in zone mass on the solution quality for the propagation of an isolated hydrodynamic discontinuity. We consider only the case of Lagrangian mode. In this problem we use the same dimensionless units with the initial density and sound speed set as $\rho=1$ and $c=1$ with a $\gamma=5 / 3$ ideal gas law equation of state everywhere. The problem is specified as a piston with speed $\mathrm{v}_{\mathrm{p}}$ in the negative $\mathrm{x}$-direction on a one dimensional domain spanning $-3<\mathrm{x}<3$ with 128 zones between $\mathrm{x}=-3$ and $\mathrm{x}=3$ and $128 \mathrm{R}$ zones between $\mathrm{x}=0$ and $\mathrm{x}=3$ where $\mathrm{R}$ is the ratio of the grid length on the upstream half of the domain to the zone length on the down stream half of the domain length (i.e. $\mathrm{R}=\mathrm{dx}_{\text {upstream }} / \mathrm{dx}_{\text {downstream }}$ ). Because the initial domain is filled with a gas of uniform density, the cell mass variation is equal to the zone length variation. Starting from this initial condition, the problem is advanced forward in time until the disturbance driven by the piston reaches $\mathrm{y}=-2.5$ as computed by the analytic value for the wave / shock propagation speed.

Neglecting the first few piston-adjacent zones that are known to be subject to the wall heating error we find that the solution error for $\mathrm{R}<1$ (a shock crossing from a finely zoned region to a coarsely zoned region) is dominated by an isolated zone of low density / high temperature and the solution error for $\mathrm{R}>1$ (a shock crossing from a coarsely zoned region to a finely zoned region) is dominated by an isolated zone of high density / low temperature. In Figure 9 we plot the relative magnitude of this error in the $\left(R, v_{p}\right)$ parameters space. The plot shows curves of logarithmically spaced variation in $v_{p}$ and the case of of $v_{p}=3$ as used in the paved mesh tests. The variation in the absolute value of the coarsening ratio $\mathrm{R}$ is spaced as a broken power law with a finer variation near $\mathrm{R}=1$. The results show a very rapid rise in the solution error about $\mathrm{R}=1$. The solution error remains below $1 \%$ for factor of 8 variation in zone spacing only for subsonic flow with $\mathrm{v}_{\mathrm{p}}<0.25$. The magnitude of the solution error plateaus for piston speeds $v p>4$. For the case of $v_{p}=3$ as used in the paved mesh problem discussed earlier, solution errors of $10 \%$ also occur for $\mathrm{R}<2 / 3$ and $\mathrm{R}>3 / 2$. The paved mesh problems considered in the previous sections do contain abrupt variations in zone mass comparable to this. Therefore the bulk of the error in the $\mathrm{L}_{\infty}$ norm in those problems can be attributed to this effect. 


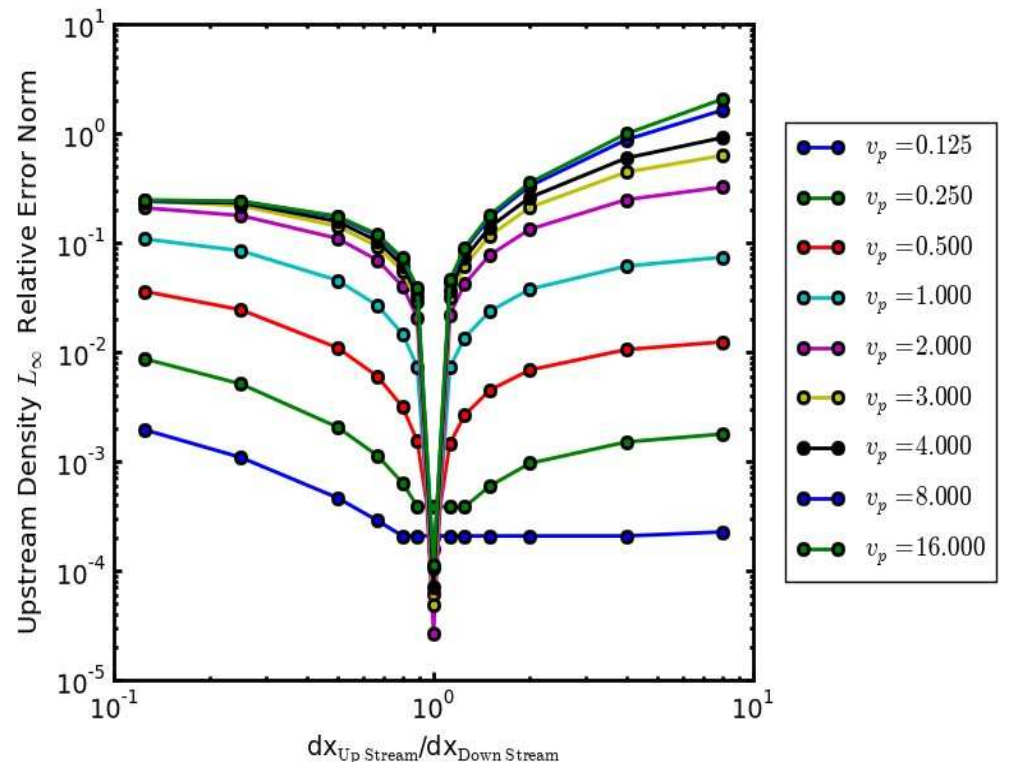

Figure 9: The maximum relative error in the density field left after the disturbance driven by an impulsive piston of speed $\mathrm{v}_{\mathrm{p}}$ has propagated across an isolated discontinuity in zone mass. The relative error is plotted as a function of the magnitude of the change in the one dimensional zone length. Because the initial condition for this test is uniform density, the change in zone mass is equal to the change in zone length.

\section{4) Conclusions}

The paving module of the Draco mesh generation code provides a unique approach to initializing HEDP problems that make use of spatially varying zone size or problems containing interfaces of arbitrary geometry. We have studied the propagation of a planar hydrodynamic shock in the Kull code through meshes that have been constructed using the Draco paver tool. We find that paved meshes do introduce errors in the post-shock state that are larger than the errors than those in the same problem with a uniform mesh that has a gradual geometric variation in zone mass. We conclude that paved meshes present a viable answer to problems containing geometries that are too complex to construct using simpler coordinate transformations with a few caveats: 1) Care must be taken to keep spatial gradients in zone mass in the paved mesh as small as possible, such as by specifying smooth and isotropic variation in the zone lengths along the perimeter of the paved region. 2) In the limit of Lagrangian mesh motion errors due to changes in zone mass remain local to these zones. Therefore, if abrupt changes in zone mass cannot be avoided, the mesh should be constructed so that these variations occur in "unimportant" regions of the domain. 3) The degree to which errors caused by zone mismatches may be tolerable for a given application will depend on the characteristic speed of the hydrodynamic disturbances under consideration. Low Mach number flows are less subject to these errors than higher Mach number flows. 